\title{
Searching for stable fullerenes in space with computational chemistry
}

\author{
Alessandra Candian, ${ }^{1 \star}$ Marina Gomes Rachid, ${ }^{1,2}$ Heather MacIsaac, $, 3,5$ \\ Viktor N. Staroverov, ${ }^{6}$ Els Peeters ${ }^{3,4,7}$ and Jan Cami ${ }^{3,4,7}$ \\ ${ }^{1}$ Leiden Observatory, Leiden University, Niels Bohrweg 2, 2333CA, Leiden, The Netherlands \\ ${ }^{2}$ Universidade do Vale do Paraíba, Laboratorio de Astroquímica e Astrobiologia, 2911, Urbanova, São José dos Campos, SP, Brazil \\ ${ }^{3}$ Department of Physics and Astronomy, The University of Western Ontario, London, ON N6A 3K7, Canada \\ ${ }^{4}$ Centre for Planetary Science and Exploration (CPSX), The University of Western Ontario, London, ON N6A 3K7, Canada \\ 5 Physics Department, St. Francis Xavier University, 5005 Chapel Square, Antigonish, NS B2G 2W5, Canada \\ ${ }^{6}$ Department of Chemistry, The University of Western Ontario, London, ON N6A 5B7, Canada \\ 7 SETI Institute, 189 Bernardo Avenue, Suite 100, Mountain View, CA 94043, USA
}

Accepted XXX. Received YYY; in original form ZZZ

\begin{abstract}
We report a computational study of the stability and infrared (IR) vibrational spectra of neutral and singly ionised fullerene cages containing between 44 and 70 carbon atoms. The stability is characterised in terms of the standard enthalpy of formation per $\mathrm{CC}$ bond, the HOMO-LUMO gap, and the energy required to eliminate $\mathrm{C}_{2}$ fragment. We compare the simulated IR spectra of these fullerene species to the observed emission spectra of several planetary nebulae (Tc 1, SMP SMC 16, and SMP LMC 56) where strong $\mathrm{C}_{60}$ emission has been detected. Although we could not conclusively identify fullerenes other than $\mathrm{C}_{60}$ and $\mathrm{C}_{70}$, our results point to the possible presence of smaller $(44,50$, and 56-atom) cages in those astronomical objects. Observational confirmation of our prediction should become possible when the James Webb Space Telescope comes online.
\end{abstract}

Key words: astrochemistry - molecular data - ISM:molecules - ISM:planetary nebulae:general - infrared:ISM

\section{INTRODUCTION}

A significant fraction $(\sim 10 \%)$ of elemental carbon in the universe is thought to exist in the form of large organic molecules such as polycyclic aromatic hydrocarbons (PAHs) and fullerenes (Tielens 2008). These species are of central importance in the physics and chemistry of interstellar environments and star-forming regions of the Milky Way and other galaxies.

Fullerenes are a fairly recent addition to the inventory of known interstellar species. Since the first detection of infrared (IR) vibrational bands of $\mathrm{C}_{60}$ and $\mathrm{C}_{70}$ in the emission of the Tc 1 planetary nebula $(\mathrm{PN})$ (Cami et al. 2010), similar IR bands have been found in many astronomical objects: various types of evolved stars (García-Hernández et al. 2010; Gielen et al. 2011; Zhang \& Kwok 2011; García-Hernández et al. 2011a,b, 2012; Evans et al. 2012; Bernard-Salas et al. 2012), reflection nebulae and HII regions (Sellgren et al. 2010;

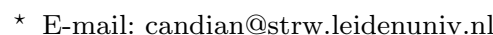

García-Hernández et al. 2012; Boersma et al. 2012; Peeters et al. 2012; Castellanos et al. 2014), young stellar objects (Roberts et al. 2012), and recently in the diffuse interstellar medium (Berné et al. 2017). Laboratory measurements and observational analyses have also shown that several diffuse interstellar bands are due to $\mathrm{C}_{60}^{+}$(Walker et al. 2015; Campbell et al. 2015, 2016; Walker et al. 2016, 2017; Cordiner et al. 2017; Lallement et al. 2018). It has thus become clear that $\mathrm{C}_{60}$ and $\mathrm{C}_{60}^{+}$ are widespread and abundant in space. Given that their unique spectral features are well characterized and understood, these species can now be used as proxies to study the much larger family of bulky aromatic species in space. .

A key question to be resolved in the context of circumstellar and interstellar fullerenes is how they are formed. Most experimental methods on Earth represent bottom-up formation routes starting from a carbonaceous seed gas (see, e.g., Jäger et al. 2009); closed network growth through intermediate size cages eventually reaches the most stable fullerene $\mathrm{C}_{60}$ (Dunk et al. 
2012). Such formation routes require high densities and thus would play out over prohibitively long timescales in astrophysical environments where densities are low (see, e.g., Micelotta et al. 2012). An important clue to the astrophysical formation routes came from the observational analysis of Berné \& Tielens (2012) who showed that the abundance of PAHs in NGC 7023 decreases as one approaches the hot central star, while at the same time the abundance of $\mathrm{C}_{60}$ increases. This suggests that fullerenes may be formed by ultraviolet photochemistry in a top-down fashion starting from large PAHs with more than 60 carbons. The chemical feasibility of such formation routes was confirmed experimentally (Zhen et al. 2014). A top-down route may explain the formation of $\mathrm{C}_{60}$ in interstellar environments; however, it is not clear whether it can also account for the formation of fullerenes in evolved stars (Cami et al. 2018).

Bottom-up routes toward $\mathrm{C}_{60}$ pass through smaller fullerene cages that are intermediate building blocks; top-down routes also involve smaller fullerene cages produced by photofragmentation of $\mathrm{C}_{60}$. Interestingly, both bottom-up (e.g. Zimmerman et al. 1991) and topdown (e.g. Rohlfing et al. 1984) routes predict enhanced abundances of so-called "magic-number" fullerenes smaller cages (with 44, 50 or 56 carbon atoms) that are more stable than other fullerenes. If these intermediate fullerenes are sufficiently stable, they may survive for long enough to be detectable in astrophysical environments.

In this paper, we calculate and analyse several structural stability indicators of various fullerenes which may be correlated with their abundance in space. We also calculate IR vibrational spectra for a representative sample of intermediate fullerenes and compare our results to astronomical observations of several $\mathrm{C}_{60}$-rich PNe.

\section{METHODOLOGY}

\subsection{Definitions}

The stability of fullerene-type molecules is determined by several factors. By Euler's polyhedron formula, a fullerene consisting only of pentagons and hexagons (classical fullerenes) must contain exactly 12 pentagons to form a closed cage. The arrangement of the pentagons affects the stability of the cage, the most stable isomer being the one where the pentagons are isolated (Kroto 1987). The buckminsterfullerene $\mathrm{C}_{60}$ and its larger cousin $\mathrm{C}_{70}$ are the smallest cages that comply with this rule (Fowler \& Manolopoulos 1995). For cages where isolated pentagons are impossible, the more stable isomers are the ones with the smallest number of adjacent pentagons (Albertazzi et al. 1999). Although the isolated pentagon rule is likely the dominant stabilisation factor for neutral and cationic cages, there are indications that it does not always work (Fowler \& Manolopoulos 1992; Wang et al. 2015).

Here, we are interested in the relative stability of smaller $\mathrm{C}_{n}$ cages with $n=44,50,56$ as well as cages that arise as intermediates in reversible transformations connecting $\mathrm{C}_{60}$ and $\mathrm{C}_{70}$, i.e., $n=62,64,66$, and 68 . Guided by the isolated pentagon rule, we selected for each $n$ the isomers containing the lowest number of adjacent pentagons in both neutral and cationic form. In the case of $\mathrm{C}_{62}$, non-classical fullerenes containing a heptagon and square were also considered, since they are expected to be more stable than the classical cages (Ayuela et al. 1996; Qian et al. 2000; Sánchez et al. 2005).

To quantify the stability of these $\mathrm{C}_{n}$ fullerene molecules, we employ three metrics. The first one is $\Delta_{f} H_{0}^{\circ}$, the standard enthalpy of formation of $\mathrm{C}_{n}$ at $T=0 \mathrm{~K}$ divided by the number of $\mathrm{CC}$ bonds. $\Delta_{f} H_{0}^{\circ}$ is defined as the change of enthalpy for the reaction in which 1 mol of $\mathrm{C}_{n}$ is formed from $n$ mol of graphite at 1 bar. Following Alcamí et al. (2007), we compute this quantity using the relation

$\Delta_{f} H_{0}^{\circ}\left(\mathrm{C}_{n}\right)=E_{0}\left(\mathrm{C}_{n}\right)-n E_{0}(\mathrm{C})+n \Delta_{f} H_{0}^{\circ}(\mathrm{C})$,

where $E_{0}\left(\mathrm{C}_{n}\right)$ is the sum of the total electronic and zero-point energies $(\mathrm{ZPE})$ of $\mathrm{C}_{n}, E_{0}(\mathrm{C})$ is the groundstate electronic energy of a gas-phase $\mathrm{C}$ atom, and $\Delta_{f} H_{0}^{\circ}(\mathrm{C})=171.29 \mathrm{kcal} / \mathrm{mol}$ is the standard enthalpy of formation of gas-phase $\mathrm{C}$ atoms at $T=0 \mathrm{~K}$. The values of $E_{0}\left(\mathrm{C}_{n}\right)$ and $E_{0}(\mathrm{C})$ were calculated with quantum chemistry techniques (see below).

Stability of fullerenes can also be described from a kinetic point of view as a measure of "resistance" to becoming an activated complex that can undergo chemical reactions such as fragmentation. A useful indicator of kinetic stability is the gap between the highest occupied molecular orbital (HOMO) and lowest unoccupied molecular orbital (LUMO) energy levels,

$\Delta \epsilon_{\mathrm{HL}}=\epsilon_{\mathrm{LUMO}}-\epsilon_{\mathrm{HOMO}}$.

A large HOMO-LUMO gap correlates with a high kinetic stability (lower chemical reactivity) of $\mathrm{C}_{n}$ molecules (Manolopoulos et al. 1991).

Finally, molecular stability can be interpreted as the enthalpy change associated with a particular fragmentation reaction. For example, it is well established that the dominant fragmentation channel of $\mathrm{C}_{n}$ cages is a sequential elimination of $\mathrm{C}_{2}$ units. The enthalpy change for this process is

$\Delta E_{0}^{\mathrm{elim}}=E_{0}\left(\mathrm{C}_{n-2}\right)+E_{0}\left(\mathrm{C}_{2}\right)-E_{0}\left(\mathrm{C}_{n}\right)$,

where the $E_{0}$ values are the ZPE-corrected total electronic energies of the indicated gas-phase species.

\subsection{Computational details}

Cartesian coordinates of all $\mathrm{C}_{n}$ cages were generated using the Fullerene software (version 4.5) (Schwerdtfeger et al. 2013), applying symmetry constraints appropriate to the corresponding point group. We optimised cage geometries and calculated their vibrational spectra with the Gaussian 09 program (Frisch et al. 2009) using the B3LYP functional (Stephens et al. 1994), the 6-31G* basis set, and a frequency scale factor $f_{\text {scale }}=0.978$. We convolved the spectra with a Lorentzian profile function having a full width at half maximum (FWHM) 
Table 1. Calculated properties of the $\mathrm{C}_{n}$ and $\mathrm{C}_{n}^{+}$fullerene cages studied in this work. All values were obtained at the B3LYP/6-31G* level of theory except for the HOMO-LUMO gap (PBE/6-31G*).

\begin{tabular}{|c|c|c|c|c|c|c|c|c|c|c|}
\hline \multirow[b]{2}{*}{$n$} & \multicolumn{6}{|c|}{ Neutral $C_{n}$} & \multicolumn{4}{|c|}{ Cation $\mathrm{C}_{n}^{+}$} \\
\hline & Isomer* & $\begin{array}{l}\text { Point } \\
\text { group }\end{array}$ & $\begin{array}{c}E_{0} \\
\left(E_{h}\right)\end{array}$ & $\begin{array}{c}\Delta_{f} H_{0}^{\circ} \\
(\mathrm{kcal} / \mathrm{mol})\end{array}$ & $\begin{array}{c}\Delta \epsilon_{\mathrm{HL}} \\
(\mathrm{eV})\end{array}$ & $\begin{array}{c}\Delta E_{0}^{\text {elim }} \\
(\mathrm{eV}) \\
\end{array}$ & $\begin{array}{l}\text { Point } \\
\text { group }\end{array}$ & $\begin{array}{c}E_{0} \\
\left(E_{h}\right)\end{array}$ & $\begin{array}{c}\Delta_{f} H_{0}^{\circ} \\
(\mathrm{kcal} / \mathrm{mol})\end{array}$ & $\begin{array}{c}\Delta E_{0}^{\mathrm{elim}} \\
(\mathrm{eV})\end{array}$ \\
\hline \multirow[t]{7}{*}{44} & 75 & $D_{2}$ & -1675.934983 & 823.3 & 0.76 & 8.51 & $C_{2}$ & -1675.675080 & 986.3 & 8.51 \\
\hline & 89 & $D_{2}$ & -1675.933854 & 824.0 & 0.84 & 8.48 & $D_{2}$ & -1675.672880 & 987.7 & 8.45 \\
\hline & 72 & $D_{3 h}$ & -1675.922892 & 830.8 & 1.23 & 8.18 & $C_{1}$ & -1675.659056 & 996.4 & 8.07 \\
\hline & 69 & $C_{1}$ & -1675.905136 & 842.0 & 0.57 & 7.70 & $C_{1}$ & -1675.651777 & 1001.0 & 7.87 \\
\hline & 87 & $C_{2}$ & -1675.890008 & 851.4 & 0.61 & 7.29 & $C_{2}$ & -1675.640556 & 1008.0 & 7.57 \\
\hline & 78 & $C_{1}$ & -1675.887802 & 852.9 & 0.43 & 7.23 & $C_{1}$ & -1675.640151 & 1008.3 & 7.56 \\
\hline & 88 & $C_{1}$ & -1675.887805 & 852.9 & 0.43 & 7.23 & $C_{1}$ & -1675.639885 & 1008.4 & 7.55 \\
\hline \multirow[t]{7}{*}{50} & 270 & $D_{3}$ & -1904.622648 & 840.7 & 1.33 & 9.57 & $C_{2}$ & -1904.360527 & 1014.3 & 9.21 \\
\hline & 271 & $D_{5 h}$ & -1904.614508 & 845.8 & 0.41 & 9.35 & $C_{5 h}$ & -1904.372567 & 997.6 & 9.54 \\
\hline & 266 & $C_{s}$ & -1904.610068 & 848.6 & 0.92 & 9.23 & $C_{s}$ & -1904.357248 & 1007.2 & 9.12 \\
\hline & 263 & $C_{2}$ & -1904.606111 & 851.1 & 1.13 & 9.13 & $C_{2}$ & -1904.344217 & 1015.4 & 8.77 \\
\hline & 264 & $C_{s}$ & -1904.594589 & 858.3 & 0.78 & 8.81 & $C_{2}$ & -1904.342077 & 1016.8 & 8.71 \\
\hline & 260 & $C_{2}$ & -1904.587465 & 862.8 & 0.54 & 8.62 & $C_{s}$ & -1904.340925 & 1017.5 & 8.68 \\
\hline & 262 & $C_{s}$ & -1904.584073 & 864.9 & 0.58 & 8.53 & $C_{s}$ & -1904.334007 & 1021.8 & 8.49 \\
\hline \multirow[t]{4}{*}{56} & 916 & $D_{2}$ & -2133.263470 & 887.6 & 0.68 & 8.36 & $C_{s}$ & -2133.019324 & 1040.8 & 8.31 \\
\hline & 864 & $C_{s}$ & -2133.263101 & 887.8 & 0.88 & 8.35 & $C_{s}$ & -2133.017361 & 1042.0 & 8.54 \\
\hline & 843 & $C_{2}$ & -2133.257250 & 891.5 & 0.70 & 8.19 & $C_{2}$ & -2133.012619 & 1045.0 & 8.13 \\
\hline & 913 & $C_{2 v}$ & -2133.252127 & 894.7 & 0.50 & 8.05 & $C_{2 v}$ & -2133.011996 & 1045.4 & 8.11 \\
\hline 60 & & $I_{h}$ & -2285.799255 & 850.7 & 1.67 & 11.18 & $D_{5 d}$ & -2285.538791 & 1014.1 & 10.47 \\
\hline \multirow[t]{4}{*}{62} & $1 \mathrm{~h}^{\dagger}$ & $C_{s}$ & -2361.916462 & 926.8 & 0.50 & 5.52 & $C_{s}$ & -2361.681847 & 1074.0 & 6.22 \\
\hline & $1 \mathrm{~s}^{\ddagger}$ & $C_{2 v}$ & -2361.910879 & 930.3 & 0.84 & 5.36 & $C_{2 v}$ & -2362.670169 & 1081.3 & 5.90 \\
\hline & 2378 & $C_{2}$ & -2361.895289 & 940.1 & 0.42 & 4.93 & $C_{1}$ & -2361.655576 & 1090.5 & 5.50 \\
\hline & 2377 & $C_{1}$ & -2361.894581 & 940.5 & 0.34 & 4.92 & $C_{2}$ & -2361.653374 & 1091.5 & 5.44 \\
\hline \multirow[t]{3}{*}{64} & 3451 & $D_{2}$ & -2438.155785 & 926.3 & 1.23 & 8.84 & $D_{2}$ & -2437.907149 & 1082.3 & 9.17 \\
\hline & 3452 & $C_{s}$ & -2438.145459 & 932.7 & 1.06 & 8.56 & $C_{s}$ & -2437.894842 & 1090.0 & 8.83 \\
\hline & 3457 & $C_{2}$ & -2438.135884 & 938.8 & 0.86 & 8.30 & $C_{2}$ & -2437.885889 & 1095.6 & 8.59 \\
\hline \multirow[t]{3}{*}{66} & 4466 & $C_{s}$ & -2514.356701 & 949.8 & 0.95 & 7.79 & $C_{2 v}$ & -2514.113257 & 1102.6 & 7.93 \\
\hline & 4348 & $C_{2 v}$ & -2514.350102 & 954.0 & 0.30 & 7.61 & $C_{s}$ & -2514.106888 & 1106.6 & 7.76 \\
\hline & 4169 & $C_{2}$ & -2514.342759 & 958.6 & 0.48 & 7.41 & $C_{2}$ & -2514.099333 & 1111.3 & 7.55 \\
\hline \multirow[t]{8}{*}{68} & 6290 & $C_{2}$ & -2590.574909 & 962.6 & 1.37 & 8.26 & $C_{2}$ & -2590.323827 & 1120.1 & 8.06 \\
\hline & 6328 & $C_{2}$ & -2590.572785 & 963.9 & 0.93 & 8.21 & $C_{2}$ & -2590.324464 & 1119.7 & 8.07 \\
\hline & 6270 & $C_{1}$ & -2590.557981 & 973.2 & 1.05 & 7.80 & $C_{1}$ & -2590.313048 & 1126.9 & 7.76 \\
\hline & 6198 & $C_{1}$ & -2590.555998 & 974.4 & 0.41 & 7.75 & $C_{1}$ & -2590.317355 & 1124.2 & 7.88 \\
\hline & 6148 & $C_{1}$ & -2590.554346 & 975.5 & 0.36 & 7.71 & $C_{1}$ & -2590.321569 & 1121.6 & 7.99 \\
\hline & 6146 & $C_{2}$ & -2590.554036 & 975.7 & 0.07 & 7.69 & $C_{2}$ & -2590.315973 & 1125.1 & 7.84 \\
\hline & 6195 & $C_{2}$ & -2590.553849 & 975.8 & 0.42 & 7.70 & $C_{2}$ & -2590.328153 & 1117.4 & 8.17 \\
\hline & 6094 & $C_{s}$ & -2590.553605 & 975.9 & 0.04 & 7.68 & $C_{s}$ & -2590.319309 & 1123.0 & 7.93 \\
\hline 70 & & $D_{5 h}$ & -2666.865607 & 929.8 & 1.70 & 10.24 & $C_{2 v}$ & -2667.046354 & 1089.6 & 10.02 \\
\hline
\end{tabular}

*Isomer identification number according to Fowler \& Manolopoulos 1995.

${ }^{\dagger}$ Structure with a heptagon.

${ }^{\ddagger}$ Structure with a square.

of $10 \mathrm{~cm}^{-1}$, unless noted otherwise. We used Gabedit (version 2.5.0; Allouche 2011) to visualize the cage structures and their vibrational modes. We chose the B3LYP functional because it had been successfully used in the past to obtain the IR spectra of other fullerenes (Adjizian et al. 2016) and conjugated hydrocarbons (Langhoff 1996) with various basis sets (4-31G, 6-31G, 6-31G*, 6-31G**, 6-311G). The B3LYP/6-31G* combination with the above frequency scaling factor provides the best agreement with experiment (Frum et al. 1991; Kern et al. 2013; Nemes et al. 1994), similar to that of the B3LYP/6-311G method used by Adjizian et al. (2016). The $\mathrm{C}_{2}$ elimination energy was also calculated at the B3LYP/6-31G* level. For each cage $\mathrm{C}_{n}$, we used the most stable isomer of $\mathrm{C}_{n-2}$ as the fragmentation product. The B3LYP/6-31G* method predicts the $\mathrm{C}_{2}$ molecule to have a triplet ground state (Díaz-Tendero et al. 2003), which is the state we used here for computing $E_{0}\left(\mathrm{C}_{2}\right)$ in Eq. (3).

Because the B3LYP functional performs poorly for HOMO-LUMO gaps of $\mathrm{C}_{60}$ (Kremer et al. 1993), we followed Beu et al. (2005) and calculated orbital energies using the PBE functional (Perdew et al. 1996) with the same $6-31 \mathrm{G}^{*}$ basis set, at the $\mathrm{PBE} / 6-31 \mathrm{G}^{*}$ geometries. 


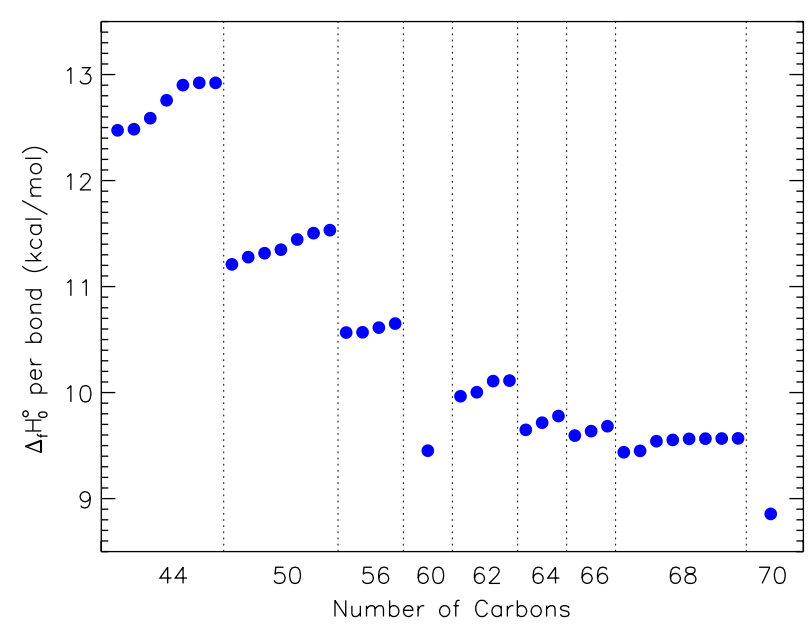

Figure 1. Standard enthalpies of formation per CC bond for neutral $\mathrm{C}_{n}$ cages, calculated with the B3LYP/6-31G* method. For each $n$, the isomers are ordered by their increasing total energy (as in Table 1). Dashed vertical lines separate the species with a different number of carbon atoms.

\section{RESULTS}

\subsection{Thermochemical stability}

We summarise the results of all thermochemical calculations of this work in Table 1; Figs. 1-3 provide a graphical representation of the same results.

Figure 1 shows the calculated enthalpy of formation per $\mathrm{CC}$ bond (assuming $3 n / 2$ bonds in $\mathrm{C}_{n}$ ) of the neutral $\mathrm{C}_{n}$ cages considered. The results are in excellent agreement with a previous study (Alcamí et al. 2007). As expected, $\mathrm{C}_{60}$ and $\mathrm{C}_{70}$ are the most stable structures according to this metric, but we note that several isomers of $\mathrm{C}_{68}$ are almost as stable as $\mathrm{C}_{60}$. The general decrease in $\Delta_{f} H_{0}^{\circ}$ (per bond) values observed from $\mathrm{C}_{40}$ to $\mathrm{C}_{70}$ correlates with the decreasing geometric distortion of the trigonal planar geometry preferred by each $s p^{2}$-hybridised carbon. Variations of the $\Delta_{f} H_{0}^{\circ}$ values among isomers are small, especially for larger cages.

Figure 2 shows the calculated HOMO-LUMO gaps for neutral $\mathrm{C}_{n}$ cages. $\mathrm{C}_{60}$ and $\mathrm{C}_{70}$ have the largest gaps among the structures examined: $1.67 \mathrm{eV}$ and $1.71 \mathrm{eV}$, respectively. The smallest gaps are close to zero $(0.04$ and $0.07 \mathrm{eV}$ for isomers 6094 and 6146 of $\mathrm{C}_{68}$ ). Note that, among the isomers with a given $n$, the structures with the largest HOMO-LUMO gaps are not always the most thermodynamically stable ones. Overall, the scatter of the $\Delta \epsilon_{\mathrm{HL}}$ values is considerably greater than that of the standard enthalpies of formation per bond, which suggests that kinetic and thermodynamic stabilities of $\mathrm{C}_{n}$ cages are not strongly correlated.

Figure 3 shows the $\mathrm{C}_{2}$ elimination energies of $\mathrm{C}_{n}$ cages in their neutral and cationic forms. In line with their exceptional stability, neutral $\mathrm{C}_{60}$ and $\mathrm{C}_{70}$ require the highest energy $(\sim 11 \mathrm{eV})$ to remove a $\mathrm{C}_{2}$ unit, followed closely by their cations. These results are in agreement with previous calculations and experimental data (Díaz-Tendero et al. 2003, 2006, and references therein).

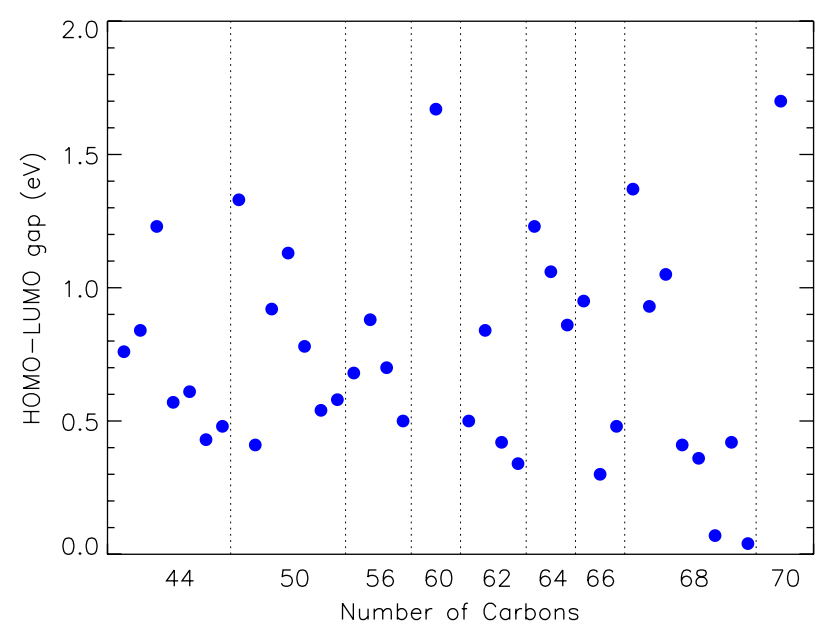

Figure 2. The HOMO-LUMO gaps of neutral $\mathrm{C}_{n}$ cages, calculated with the PBE/6-31G* method. The isomers are ordered and separated as in Fig. 1.

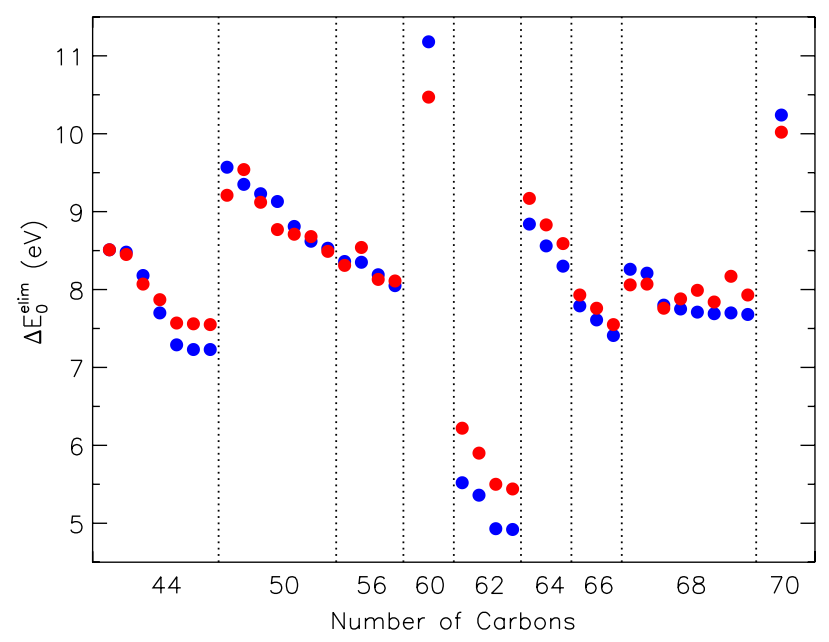

Figure 3. $\mathrm{C}_{2}$ elimination energies of various $\mathrm{C}_{n}$ (blue circles) and $\mathrm{C}_{n}^{+}$cages (red circles) calculated by the B3LYP/6-31G* method using Eq. (3). For each $n$, the isomers are ordered by their increasing total energy. The order of stability for the $\mathrm{C}_{n}^{+}$cages is the same as for corresponding neutral fullerenes with the exception of $n=66$ and 68 (see Table 1). Dashed vertical lines are drawn to guide the eye.

The next most stable cages according to this metric are $\mathrm{C}_{50}$ and $\mathrm{C}_{64}$, while the $\mathrm{C}_{62}$ isomers are the least stable. This ordering is preserved for the respective cations. Generally, $\mathrm{C}_{2}$ elimination energies of the $\mathrm{C}_{n}^{+}$cations are close to the $\Delta E_{0}^{\text {elim }}$ values of the corresponding neutral $\mathrm{C}_{n}$ structures.

\subsection{Infrared spectra}

The full collection of the simulated spectra of the fullerenes of Table 1 is displayed in Figs. 4-8. Machinereadable tables with the calculated spectra are available 

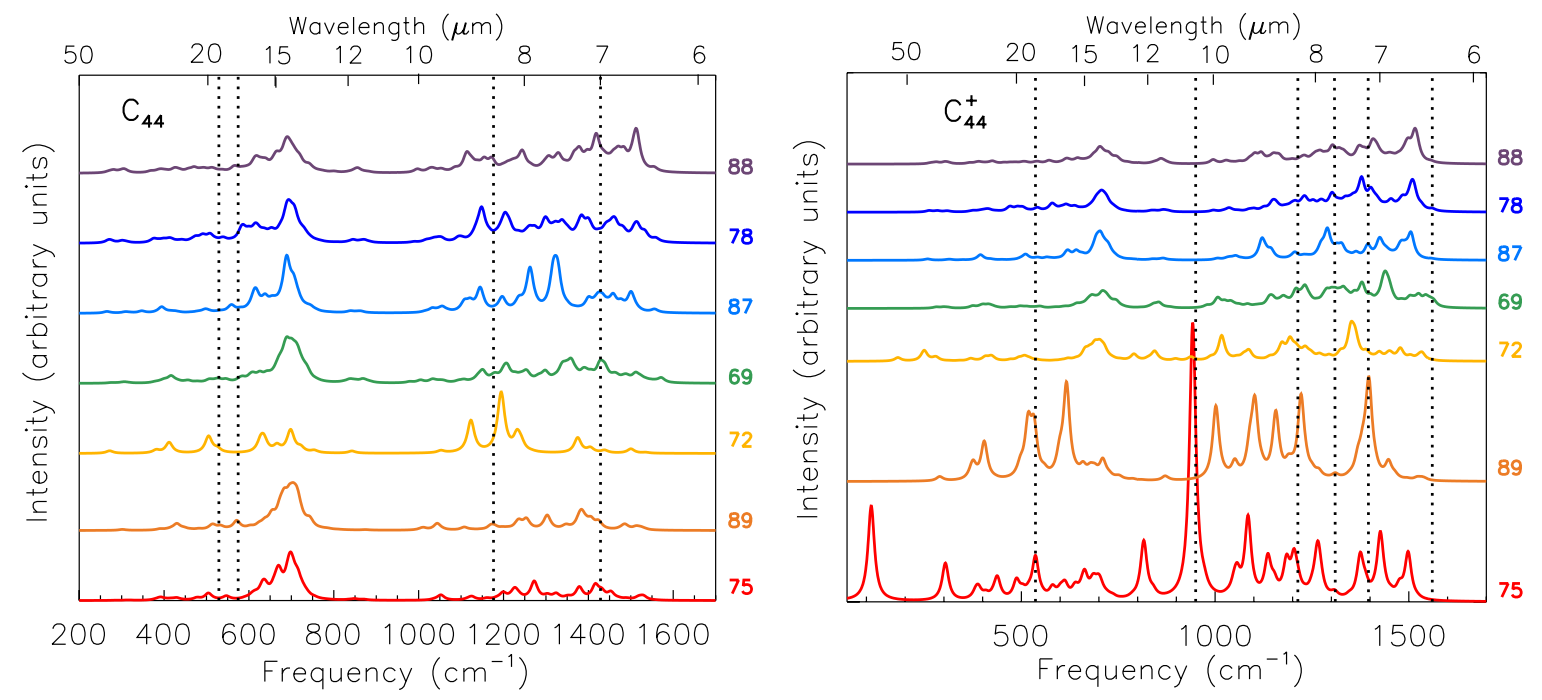

Figure 4. Simulated IR absorption spectra of various isomers of neutral $\mathrm{C}_{44}$ (left panel) and of their cationic forms (right panel). The spectra are ordered from top to bottom as in Table 1. The dotted lines show the positions of the IR-active modes of $\mathrm{C}_{60}$ at $7.0,8.5,17.4$, and $18.9 \mu \mathrm{m}$ (left) and $\mathrm{C}_{60}^{+}$at $6.4,7.1,7.5,8.2,10.4$, and $18.9 \mu \mathrm{m}$ (right).
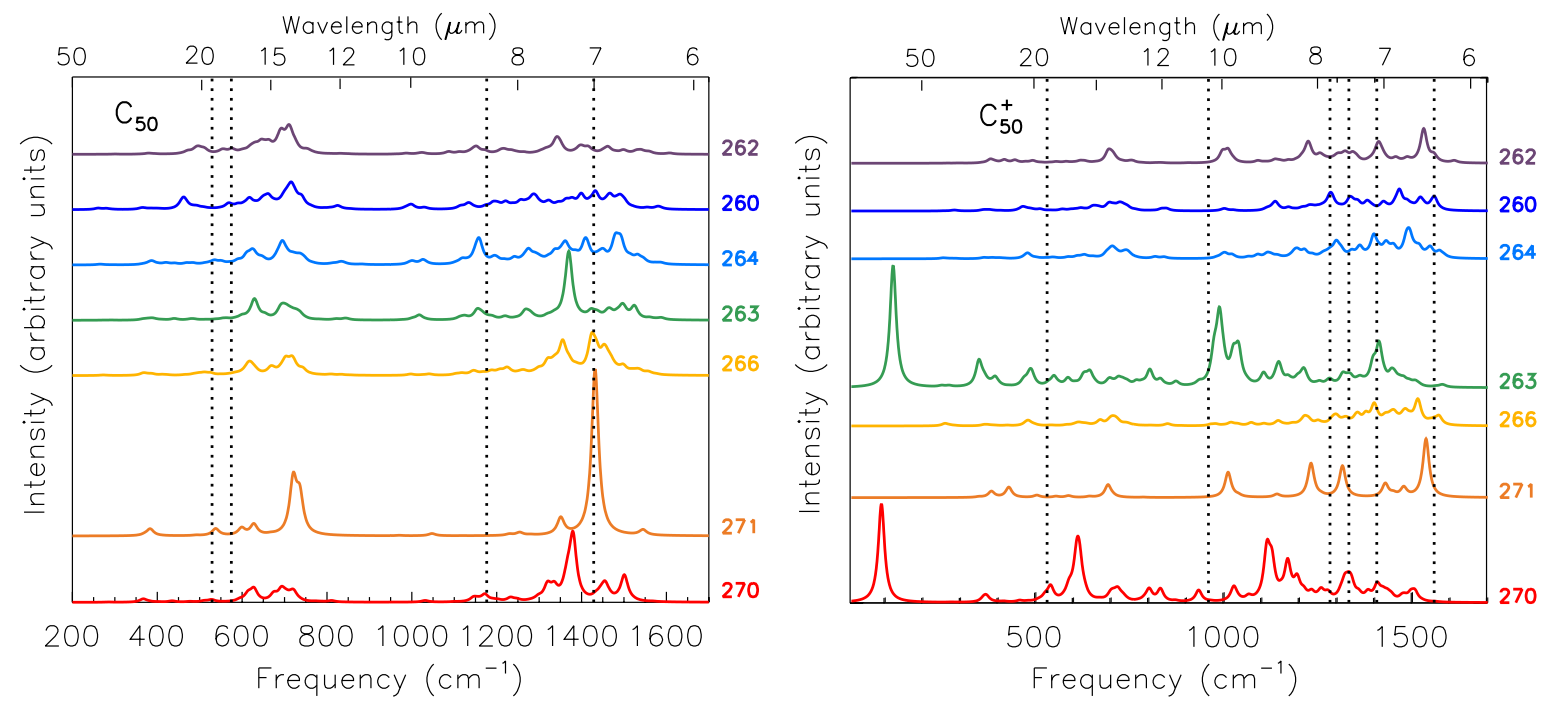

Figure 5. Same as in Fig. 4 but for $\mathrm{C}_{50}$ (left panel) and of their cationic forms (right panel).

as Supplementary Material. At first glance, IR spectra of all neutral cages display many similarities. Most of the IR-active modes cluster in two spectral regions: $1540-1000 \mathrm{~cm}^{-1}(6.5-10 \mu \mathrm{m})$ for CC stretching modes and $800-550 \mathrm{~cm}^{-1}(12.5-18.2 \mu \mathrm{m})$ for carbon-skeleton breathing modes. This is consistent with the calculated spectra of smaller cages $(n=20,24,26,28$, and 30$)$ reported by Adjizian et al. (2016). The extent of the first spectral region is almost constant within our set of spectra, while the second is subject to variations. This is a consequence of the fact that the breathing modes are much more specific to each species; therefore, this wavelength range can be considered as a "fingerprint region" for individual species.

In several cases, IR active modes show up around $1000-900 \mathrm{~cm}^{-1}(10.0-11.1 \mu \mathrm{m})$, due to combinations of CC stretching and cage-breathing modes (see, for example, $\mathrm{C}_{50}$ and $\mathrm{C}_{68}$ in Figs. 5 and 8). No strong or moderately strong IR-active modes are found below $200 \mathrm{~cm}^{-1}(50 \mu \mathrm{m})$ and above $1600 \mathrm{~cm}^{-1}(6.25 \mu \mathrm{m})$. The detailed appearance of the IR spectrum depends on the specific molecular structure and is influenced by symmetry: the lower the symmetry, the larger the number of low-intensity IR modes. This effect is exemplified by $\mathrm{C}_{50}$ isomers $271\left(D_{5 h}\right)$ and $260\left(C_{2}\right)$ in the left panel of Fig. 5 - the isomer 271 has a much higher symmetry than the isomer 260 and consequently exhibits only a few strong bands, whereas the isomer 260 shows a large number of weaker bands.

Neutral cages generally have the strongest IR transitions in the $665-714 \mathrm{~cm}^{-1}$ region $(15.0-14.0 \mu \mathrm{m})$. Even when the strongest transition is not in this range, 

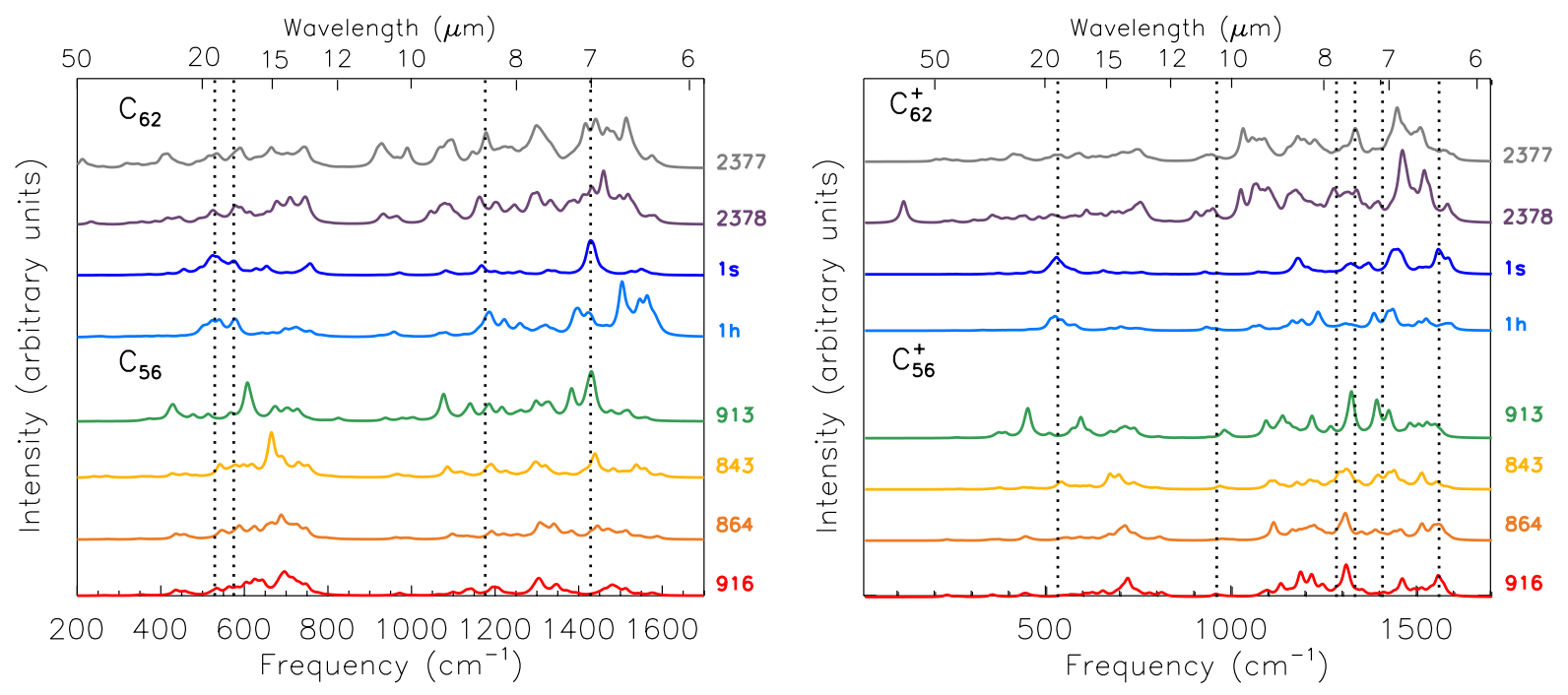

Figure 6. Same as in Fig. 4 but for $\mathrm{C}_{56}$ and $\mathrm{C}_{62}$ (left panel) and of their cationic forms (right panel).
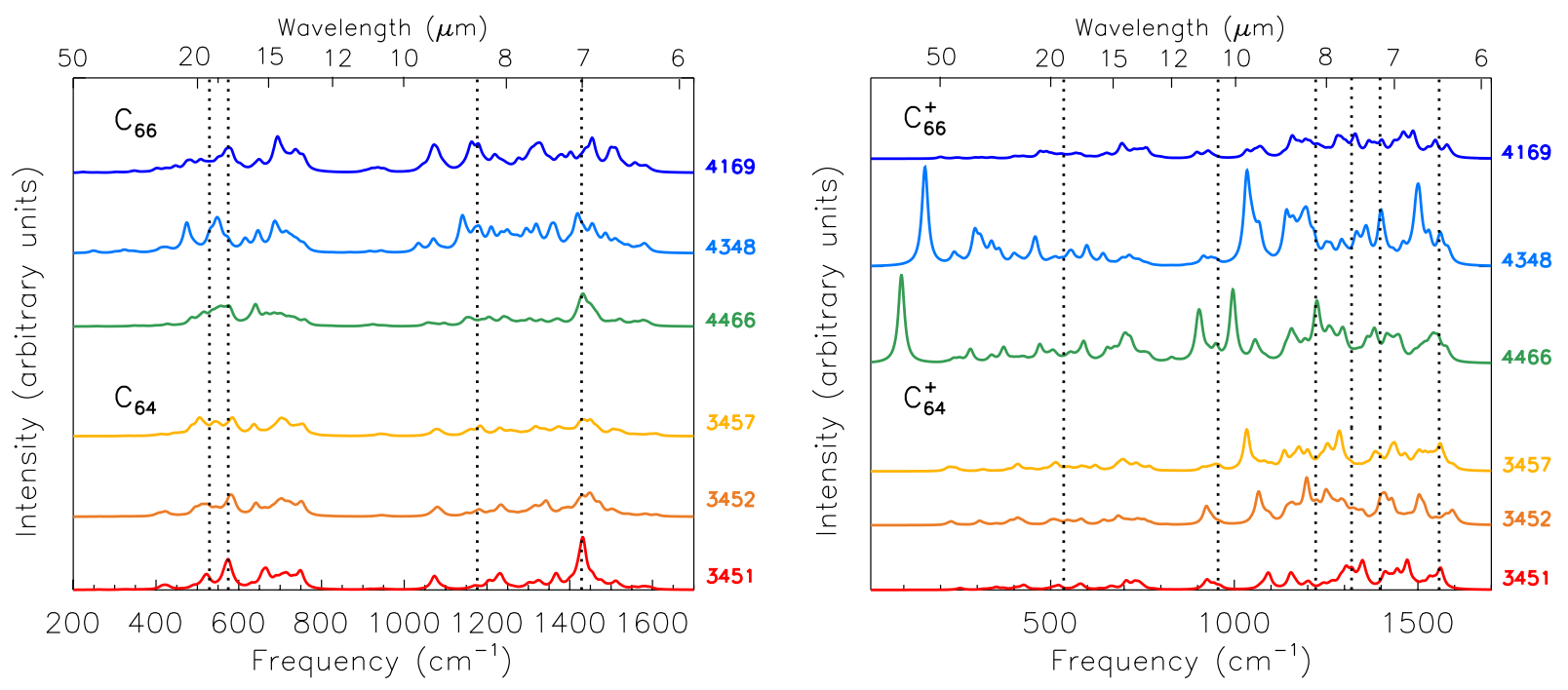

Figure 7. Same as in Fig. 4 but for $\mathrm{C}_{64}$ and $\mathrm{C}_{66}$ (left panel) and of their cationic forms (right panel).

it always contains a group of superposed mediumintensity transitions, creating broad peaks above what appears as a low-intensity plateau. Some notable exceptions are the highly symmetric $\mathrm{C}_{50}$ isomer 270 $\left(D_{5 h}\right)$ and $\mathrm{C}_{70}\left(D_{5 h}\right)$, all showing their strongest modes around $7 \mu \mathrm{m}\left(1430 \mathrm{~cm}^{-1}\right)$. Other low-symmetry neutral cages may show strong modes between 1540 and $1000 \mathrm{~cm}^{-1}$, but then the integrated intensity in this region is comparable to that of the breathing-mode region.

Few small cages $(n<60)$, but almost all of the larger ones, show strong modes close to the characteristic frequencies of $\mathrm{C}_{60}: 7.0,8.5,17.4$, and $18.9 \mu \mathrm{m}$. For isomer 271 of $\mathrm{C}_{50}$ and for $\mathrm{C}_{70}$, the band at $7.0 \mu \mathrm{m}$ is the strongest in their spectra.

The spectra of the ionised cages resemble those of their neutral counterparts, but generally show enhanced CC stretching modes $\left(1540-1000 \mathrm{~cm}^{-1}\right.$ or $\left.6.6-10 \mu \mathrm{m}\right)$.
Comparing the calculated absolute intensities for neutral and ionised fullerene, one sees that this effect is due to a decrease of the intensity of the skeleton-breathing modes at longer wavelength. Moreover, there is a tendency for the modes to move to shorter wavelength (Adjizian et al. 2016). The effect of ionisation in fullerene thus mirrors what is seen in other large conjugated system such as PAHs (Bauschlicher et al. 2008, e.g.).

Highly symmetric neutral $\mathrm{C}_{n}$ cages have degenerate but completely filled HOMOs, so they form nondegenerate ground states. After the removal of an electron, the HOMO-level degeneracy comes into play and the ionised cages spontaneously lower their symmetry due to the Jahn-Teller distortion (Jahn \& Teller 1937). C 60 is particularly notable in that regard (Berné et al. 2013; Kern et al. 2013). The lower symmetry increases the complexity of the cation spectrum, as seen for instance in isomer 271 of $\mathrm{C}_{50}^{+}$(Fig. 5). In a few cases (including 

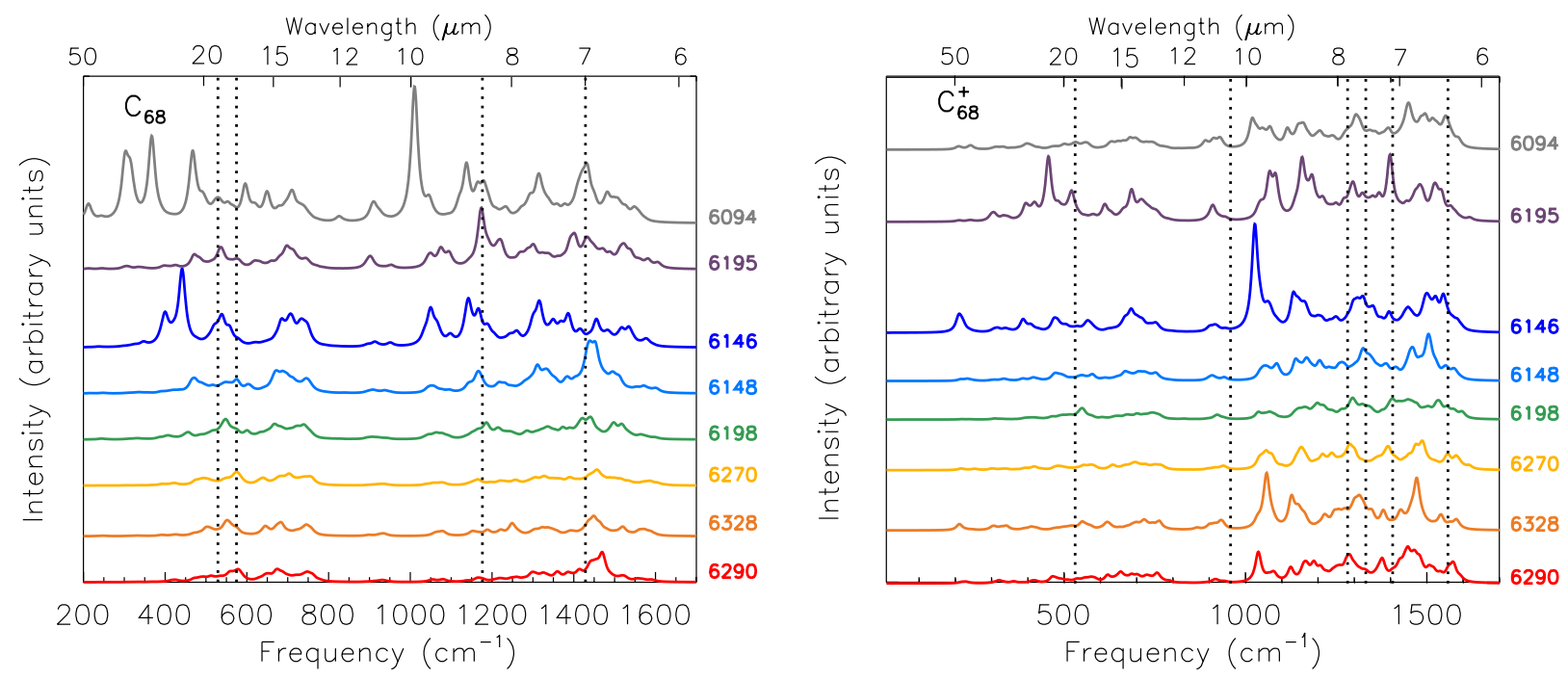

Figure 8. Same as in Fig. 4 but for $\mathrm{C}_{68}$ (left panel) and of their cationic forms (right panel).

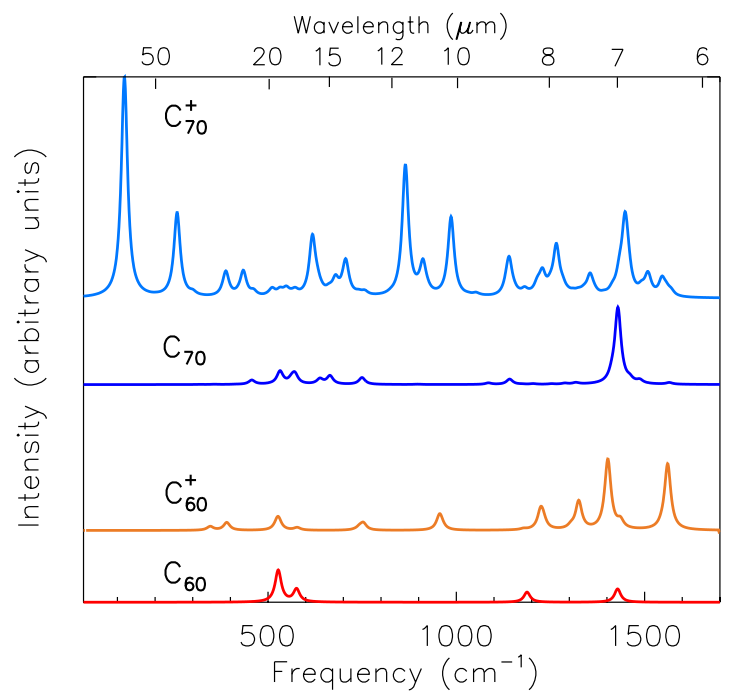

Figure 9. IR spectra of $\mathrm{C}_{60}$ and $\mathrm{C}_{70}$ in neutral and cationic forms calculated with the B3LYP/6-31G* method $\left(f_{\text {scale }}=0.978\right)$,

isomer 75 of $\mathrm{C}_{44}^{+}, \mathrm{C}_{66}^{+}$, and $\mathrm{C}_{70}^{+}$), the $\mathrm{IR}$ spectrum predicted by B3LYP/6-31G* shows anomalously high IR intensities, often for very low energy modes, as well as a complete mismatch with the spectra of their neutral counterparts (see Section 3.3 for further discussion of this aspect).

Finally, in our sample we have two non-classical isomers, a $\mathrm{C}_{62}$ isomer containing a square 4-membered ring (1s) and a $\mathrm{C}_{62}$ isomer containing a 7-membered ring (1h). For isomer 1s, the spectrum has only a few modes with very low intensities involving the square motif and this does not change much upon ionisation. On the other hand, isomer $1 \mathrm{~h}$ has medium-strong peaks at $1179,1222,1387$, and $1391 \mathrm{~cm}^{-1}(8.52,8.18,7.21$, and $7.19 \mu \mathrm{m}$ ) originating mostly from the stretching of the 7 -membered ring. Also, $\mathrm{C}_{62}-1 \mathrm{~h}$ is the only neutral cage in our sample showing strong signals below 1500 $\mathrm{cm}^{-1}$, at $1504 \mathrm{~cm}^{-1}(6.65 \mu \mathrm{m}), 1546 \mathrm{~cm}^{-1}(6.47 \mu \mathrm{m})$ and $1565 \mathrm{~cm}^{-1}(6.39 \mu \mathrm{m})$ (Fig. 6, left panel). While the first mode is due to a CC stretch in the two pentagons sharing bonds with the heptagons, the last two modes originate from the stretch of the CC bond connecting two hexagons opposite of the 7 -membered ring (Fig. 10). Upon ionisation, the modes involving the heptagon move slightly $\left(10-20 \mathrm{~cm}^{-1}\right)$ to higher frequencies and preserve their intensities. The modes lower than $1500 \mathrm{~cm}^{-1}(6.67 \mu \mathrm{m})$ have decreased intensities by a factor of $2-3$.

\subsection{Challenges of computing the IR spectrum of $\mathbf{C}_{70}^{+}$(and other ions)}

Bauschlicher \& Ricca (2010) documented the failure of the B3LYP functional for open-shell cationic PAHs including 5-membered rings. These authors suggested using the BP86 functional to verify the calculated fre- 


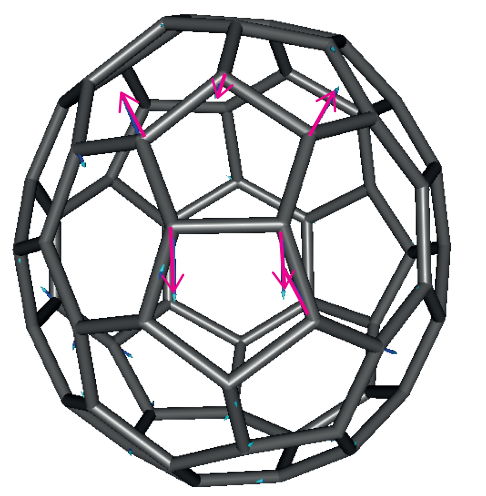

$$
\begin{aligned}
& \omega=1504 \mathrm{~cm}^{-1} \\
& I=80 \mathrm{~km} / \mathrm{mol}
\end{aligned}
$$

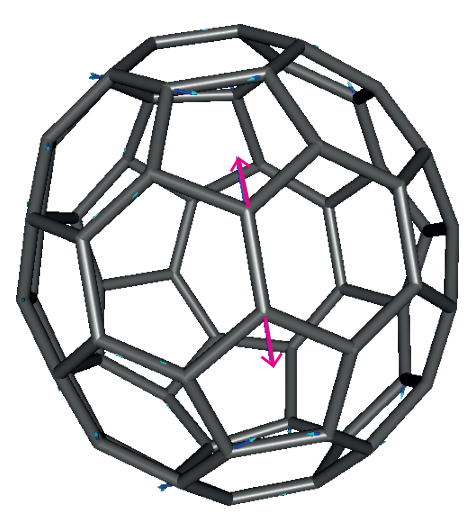

$$
\omega=1565 \mathrm{~cm}^{-1}
$$$$
\mathrm{I}=52 \mathrm{~km} / \mathrm{mol}
$$

Figure 10. Calculated normal vibrational modes associated with two intense IR peaks for isomer $1 \mathrm{~h}$ of $\mathrm{C}_{62}$. In the top image the 7 membered ring is on the right-hand side of the pentagons involved in the mode; in the bottom image the 7 -membered ring can be seen on the back CC bond involved in the mode.

quencies and intensities. We followed their suggestion and calculated the BP86/6-31G* IR spectrum of $\mathrm{C}_{70}^{+}$. Fig. 11 compares the experimental (Kern et al. 2016) and two calculated spectra of $\mathrm{C}_{70}^{+}$for the correct structure of $C_{2 v}$ symmetry. The BP86 spectrum has an intense feature at $1000 \mathrm{~cm}^{-1}$ which is not observed in the experimental spectrum. In general, positions of the other modes are in better agreement with experimental values than for the B3LYP spectrum. Kern et al. (2016) reached a similar conclusion even if the symmetry of their structure was lower $\left(C_{s}\right.$ and $C_{i}$ instead of $\left.C_{2 v}\right)$. Popov et al. (2009) calculated the vibrational frequencies of $\mathrm{C}_{70}^{+}$with the $\mathrm{PBE} / \mathrm{TZ} 2 \mathrm{P}$ methods and their results are in better agreement with our BP86/6-31G* frequencies than with the $\mathrm{B} 3 \mathrm{LYP} / 6-31 \mathrm{G}^{*}$ results. Interestingly, the strongest $\mathrm{B} 3 \mathrm{LYP} / 6-31 \mathrm{G}^{*}$ peak at $121 \mathrm{~cm}^{-1}$ corresponds to the splitting of the $E_{2}^{\prime}$ mode of $\mathrm{C}_{70}$ which arises due to the Jahn-Teller distortion of $\mathrm{C}_{70}^{+}$. Contributions from other spin multiplicity states can be ruled out, since the quadruplet lies $1.5 \mathrm{eV}$ higher than the doublet. A possible explanation of these anomalous calculated spectra may lie in the breakdown of the BornOppenheimer approximation due to the presence of excited states at energies comparable to vibrational energies. It is thus clear that, for some fullerene ions such as
$\mathrm{C}_{70}^{+}$, calculations of IR spectra based on the harmonic approximation to a single potential energy surface are unreliable and should be interpreted with great caution.

\subsection{Comparison with astronomical observations}

$\mathrm{C}_{n}$ cages with $n=44,50,56,62,64$, and 68 are likely contributors to the IR emission of the astrophysical environments known to contain significant amounts of $\mathrm{C}_{60}$. To assess the contribution of those structures, we compared their IR spectra to the observations of the three fullerene-rich PNe studied in Bernard-Salas et al. (2012): the galactic PN Tc 1, and SMP SMC 16 (SMC16 hereafter) and SMP LMC 56 (LMC56 hereafter) - similar objects in the Magellanic Clouds. These observations show $\mathrm{C}_{60}$ emission, whereas our theoretical calculations pertain to absorption spectra. The emission process for these species starts by absorption of a UV photon; this is followed by a rapid iso-energetic transition to the electronic ground state, but the process leaves the molecules in a highly excited vibrational state. Fluorescent IR emission then cools the molecule, and this is the emission that we observe. It is possible to carry out a full calculation for this IR fluorescent emission, but for our purposes we will use an approximation that is simpler and faster. Indeed, the full fluorescence spectrum can be approximated by multiplying the calculated intensities by the Planck function at a well-chosen temperature; we used $T=750 \mathrm{~K}$ (see e.g. Bauschlicher et al. 2010). A full numerical fluorescence calculation would primarily differ in providing different relative intensities between the shortest and the longest wavelength modes. Given the uncertainties associated the intrinsic IR intensities and with details of the emission mechanism, this approximation is thus justified for our purposes. We also convolved the resulting emission peaks with a Gaussian profile with a FWHM of $8 \mathrm{~cm}^{-1}$, the width of the observed $\mathrm{C}_{60}$ bands (Cami et al. 2010). Since the PNe mentioned above do not show any evidence of $\mathrm{C}_{60}^{+}$, we focus our comparison on the neutral species.

The observed IR spectra of SMC16 and LMC56 have several features in the $770-665 \mathrm{~cm}^{-1}(13-15 \mu \mathrm{m})$ region $^{1}$, where most of the $\mathrm{C}_{n}$ cages studied in this work show activity. Furthermore, the detailed structure of the peaks in the $6-8 \mu \mathrm{m}$ range of the observed spectrum is well reproduced by the summed spectra of the small cages (Fig. 12). Nevertheless, the correlation between the observed and simulated spectra is too tenuous to prove the presence of any specific $\mathrm{C}_{n}$ cage in these astronomical objects. Such assignments are made especially challenging by the fact that the abundance of non- $\mathrm{C}_{60}$ cages is expected to be much lower than that of $\mathrm{C}_{60}$; in addition, intrinsic intensities of the IR-active modes of

\footnotetext{
1 We note that there is a well-known artifact of the Spitzer IR spectrograph between 13.2 and $14 \mu \mathrm{m}$, called the "teardrop", which shows up in observations through the SL1 module; the features discussed here originate from the LL2 module and hence cannot be attributed to the teardrop artifact.
} 
Fullerenes in space

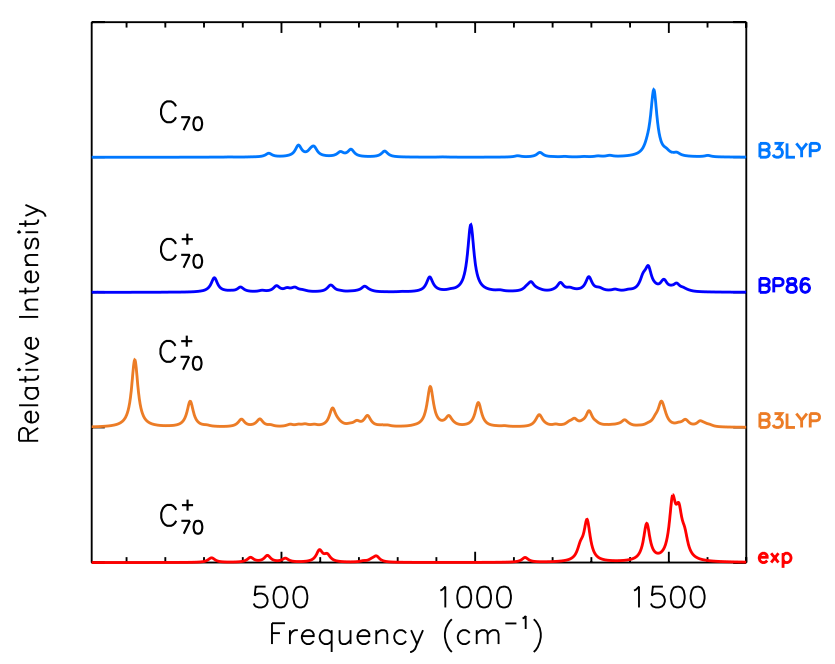

Figure 11. Comparison between the experimental IR spectrum of $\mathrm{C}_{70}^{+}$obtained by Kern et al. (2016) and theoretical IR spectra calculated with B3LYP/6-31G* and BP86/6-31G* for the correct $C_{2 v}$ structure. The B3LYP/6-31G* spectrum of neutral $\mathrm{C}_{70}$ is also shown for comparison. The spectra are convolved with a Lorentzian function with a FWHM of $10 \mathrm{~cm}^{-1}$ and normalised to the strongest feature.

other fullerenes are lower than those of $\mathrm{C}_{60}$. Thus, individual species should have very weak emission features at best.

If we cannot detect individual species, could we perhaps detect a spectral fingerprint of an entire cage population? Figure 13 shows simulated summed emission spectra of small and large $\mathrm{C}_{n}$ cages, in each case compared to the observed IR spectrum of SMC 16. The smaller cages show features in the $13-15 \mu \mathrm{m}$ region and intriguingly, the average spectrum of the smaller cages shows a weak, broad feature seen in both LMC 56 and SMC 16 without overestimating the flux at any other wavelength (Note that for a lower temperature in our emission model, the emission peaks in the 6-9 $\mu \mathrm{m}$ region would be weaker). It is thus conceivable that a population of smaller cages is indeed present in these objects. The observations are compatible with such a population.

A similar average spectrum of the most stable larger cages (64 to 70 carbon atoms) also yields a small feature in the $13-15 \mu \mathrm{m}$ region that is weaker and closer to $13 \mu \mathrm{m}$. The summed spectra also shows a mode around $7 \mu \mathrm{m}\left(1728 \mathrm{~cm}^{-1}\right)$, which can be attributed to $\mathrm{C}_{50}$ (isomer 271) and $\mathrm{C}_{70}$, and would overlap with the corresponding $\mathrm{C}_{60}$ band. Here too, the observations are compatible with a population of larger cages, but they offer no robust detection.

Finally, it is worth pointing out that the cages studied here have IR-active modes between $6-9 \mu \mathrm{m}$ corresponding to a strong plateau that is seen underneath the emission features in all three astronomical objects. A population of smaller and/or larger cages could thus also be partly responsible for this plateau emission. On the other hand, little to no emission is seen in the 10$13 \mu \mathrm{m}$ region, where another plateau appears in the observations. Therefore, this emission plateau cannot be due to fullerenes.

\section{CONCLUSIONS}

We have investigated the stability of fullerene cages with 44 to 70 carbon atoms and calculated their IR-active vibrational modes. The IR spectra of different isomers of the same species are similar, and therefore it is difficult to identify specific isomers based on their IR spectra, except perhaps for the highly symmetric species such as $\mathrm{C}_{50}$ isomer 271. The spectra of most cages that are smaller than $\mathrm{C}_{60}$ all show features in the $13-15 \mu \mathrm{m}$ range, where the astronomical spectra of fullerene-rich planetary nebulae also contain characteristic signals. We find that the astronomical observations are compatible with the presence of a population of fullerene cages, but offer no robust evidence for them. Better chances to identify these species will be possible when the James Webb Space Telescope, to be launched in 2021, will provide high-quality spectra of $\mathrm{C}_{60}$-containing objects. The theoretical spectra presented here will be useful in interpreting those data. Finally, we want to point out that the presence of low-lying electronic states for some highly symmetric fullerene ions can greatly complicate matters and that their vibrational spectra calculated in the Born-Oppenheimer and harmonic approximations should be treated with cautious distrust.

\section{ACKNOWLEDGEMENTS}

AC acknowledge financial support from the Netherlands Organisation for Scientific research (NWO) through a Veni grant (No. 639.041.543). Calculations were carried out on the Dutch national e-infrastructure (Cartesius) with the support of SURF Cooperative, under NWOEW project SH-362-15. Part of the research was performed under the Leiden/ESA Astrophysics Program for Summer Students (LEAPS). JC, EP, and VNS acknowledge support from NSERC through the Discovery 

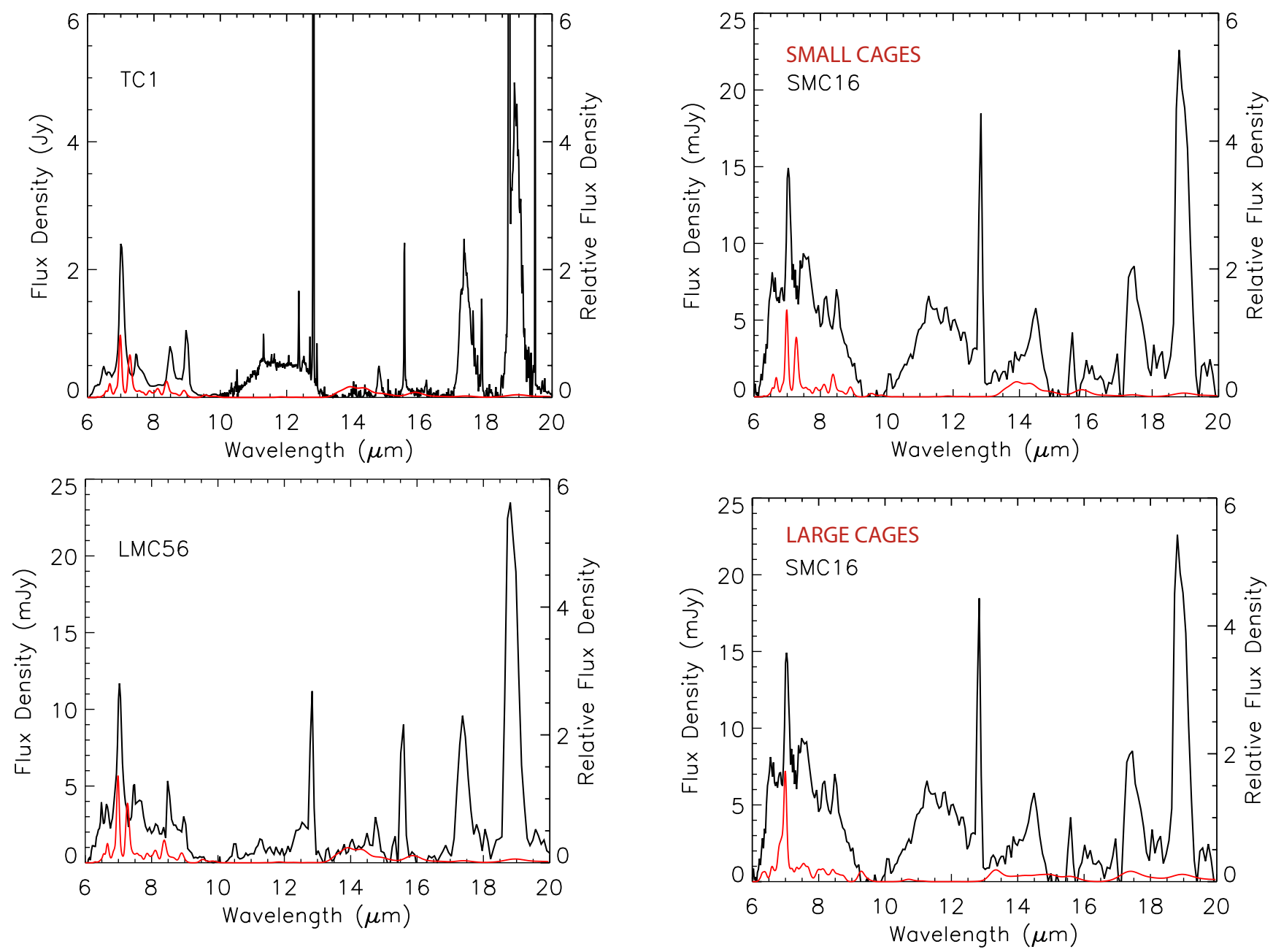

Figure 12. Observed Spitzer/IRS mid-IR spectra (black) of Tc 1 and SMC 56 (from Bernard-Salas et al. 2012) compared to the summed emission spectrum (red) of $\mathrm{C}_{44}$ (isomers $72,75,89$ ), $\mathrm{C}_{50}$ (isomers $263,270,271$ ), and $\mathrm{C}_{60}$.

Grants Program. HM was supported by an interdisciplinary undergraduate research award from the Centre for Planetary Science and Space Exploration (CPSX) at the University of Western Ontario.

Figure 13. Top panel: Observed Spitzer/IRS mid-IR spectrum of SMC 16 (black) from Bernard-Salas et al. (2012) compared to the summed emission spectrum (red) of $\mathrm{C}_{44}$ (isomers $72,75,89$ ), $\mathrm{C}_{50}$ (isomers $263,270,271$ ), and $\mathrm{C}_{60}$. Bottom panel: The same observational spectrum of SMC 16 compared to the summed emission spectrum of $\mathrm{C}_{64}$ (isomers 3451,3452 ), $\mathrm{C}_{68}$ (isomers 6290, 6328, 6270), $\mathrm{C}_{60}$, and $\mathrm{C}_{70}$.

\section{REFERENCES}

Adjizian J.-J., Vlandas A., Rio J., Charlier J.-C., Ewels C. P., 2016, Philos. Trans. Roy. Soc. A, 374, 20150323

Albertazzi E., Domene C., Fowler P. W., Heine T., Seifert G., Van Alsenoy C., Zerbetto F., 1999, Phys. Chem. Chem. Phys., 1, 2913

Alcamí M., Sánchez G., Díaz-Tendero S., Wang Y., Martín F., 2007, J. Nanosci. Nanotechnol., 7, 1329

Allouche A.-R., 2011, J. Comput. Chem., 32, 174

Ayuela A., Fowler P. W., Mitchell D., Schmidt R., Seifert G., Zerbetto F., 1996, J. Phys. Chem., 100, 15634

Bauschlicher C. W., Ricca A., 2010, Mol. Phys., 108, 2647

Bauschlicher Jr. C. W., Peeters E., Allamandola L. J., 2008, ApJ, 678,316

Bauschlicher Jr. C. W., et al., 2010, ApJS, 189, 341

Bernard-Salas J., Cami J., Peeters E., Jones A. P., Micelotta 
E. R., Groenewegen M. A. T., 2012, ApJ, 757, 41

Berné O., Tielens A. G. G. M., 2012, Proc. Nat. Acad. Sci. USA, 109, 401

Berné O., Mulas G., Joblin C., 2013, A\&A, 550, L4

Berné O., Cox N. L. J., Mulas G., Joblin C., 2017, A\&A, 605, L1

Beu T. A., Onoe J., Hida A., 2005, Phys. Rev. B, 72, 155416

Boersma C., Rubin R. H., Allamandola L. J., 2012, ApJ, 753, 168

Cami J., Bernard-Salas J., Peeters E., Malek S. E., 2010, Science, 329, 1180

Cami J., Peeters E., Bernard-Salas J., Doppmann G., De Buizer J., 2018, Galaxies, 6, 101

Campbell E. K., Holz M., Gerlich D., Maier J. P., 2015, Nature, 523,322

Campbell E. K., Holz M., Maier J. P., Gerlich D., Walker G. A. H., Bohlender D., 2016, ApJ, 822, 17

Castellanos P., Berné O., Sheffer Y., Wolfire M. G., Tielens A. G. G. M., 2014, ApJ, 794, 83

Cordiner M. A., et al., 2017, ApJ, 843, L2

Díaz-Tendero S., Alcamí M., Martín F., 2003, J. Chem. Phys., 119,5545

Díaz-Tendero S., Sánchez G., Alcamí M., Martín F., 2006, Int. J Mass Spectr., 252, 133

Dunk P. W., et al., 2012, Nat. Commun., 3, 855

Evans A., et al., 2012, MNRAS, 421, L92

Fowler P., Manolopoulos D., 1992, Nature, 355, 428

Fowler P., Manolopoulos D., 1995, An Atlas of Fullerenes. Oxford: Clarendon press

Frisch M. J., et al., 2009, Gaussian 09, Revision D.01

Frum C. I., Engleman Jr. R., Hedderich H. G., Bernath P. F., Lamb L. D., Huffman D. R., 1991, Chem. Phys. Lett., 176, 504

García-Hernández D. A., Manchado A., García-Lario P., Stanghellini L., Villaver E., Shaw R. A., Szczerba R., PereaCalderón J. V., 2010, ApJ, 724, L39

García-Hernández D. A., Kameswara Rao N., Lambert D. L., 2011a, ApJ, 729, 126

García-Hernández D. A., et al., 2011b, ApJ, 737, L30

García-Hernández D. A., Villaver E., García-Lario P., AcostaPulido J. A., Manchado A., Stanghellini L., Shaw R. A., Cataldo F., 2012, ApJ, 760, 107

Gielen C., Cami J., Bouwman J., Peeters E., Min M., 2011, A\&A, 536, A54

Jäger C., Huisken F., Mutschke H., Jansa I. L., Henning T., 2009, ApJ, 696, 706

Jahn H. A., Teller E., 1937, Proc. Roy. Soc. A, 161, 220

Kern B., Strelnikov D., Weis P., Böttcher A., Kappes M. M., 2013, J. Phys. Chem. A, 117, 8251

Kern B., Böttcher A., Strelnikov D., 2016, J. Phys. Chem. A, 120, 5868

Kremer R. K., Rabenau T., Maser W. K., Kaiser M., Simon A., Haluška M., Kuzmany H., 1993, Appl. Phys. A, 56, 211

Kroto H. W., 1987, Nature, 329, 529

Lallement R., et al., 2018, A\&A, 614, A28

Langhoff S. R., 1996, J. Phys. Chem., 100, 2819

Manolopoulos D. E., May J. C., Down S. E., 1991, Chem. Phys. Lett., 181, 105

Micelotta E. R., Jones A. P., Cami J., Peeters E., Bernard-Salas J., Fanchini G., 2012, ApJ, 761, 35

Nemes L., Ram R. S., Bernath P. F., Tinker F. A., Zumwalt M. C., Lamb L. D., Huffman D. R., 1994, Chem. Phys. Lett., 218, 295

Peeters E., Tielens A. G. G. M., Allamandola L. J., Wolfire M. G., 2012, ApJ, 747, 44

Perdew J. P., Burke K., Ernzerhof M., 1996, Phys. Rev. Lett., 77, 3865

Popov A. A., Burtsev A. V., Senyavin V. M., Dunsch L., Troyanov S. I., 2009, J. Phys. Chem. A, 113, 263
Qian W., Bartberger M. D., Pastor S. J., Houk K. N., Wilkins C. L., Rubin Y., 2000, J. Am. Chem. Soc., 122, 8333

Roberts K. R. G., Smith K. T., Sarre P. J., 2012, MNRAS, 421, 3277

Rohlfing E. A., Cox D. M., Kaldor A., 1984, J. Chem. Phys., 81, 3322

Sánchez G., Díaz-Tendero S., Alcamí M., Fernando M., 2005, Chem. Phys. Lett., 416, 14

Schwerdtfeger P., Wirz L., Avery J., 2013, J. Comput. Chem., 34, 1508

Sellgren K., Werner M. W., Ingalls J. G., Smith J. D. T., Carleton T. M., Joblin C., 2010, ApJ, 722, L54

Stephens P. J., Devlin F. J., Chabalowski C. F., Frisch M. J., 1994, J. Phys. Chem., 98, 11623

Tielens A. G. G. M., 2008, ARA\&A, 46, 289

Walker G. A. H., Bohlender D. A., Maier J. P., Campbell E. K., 2015, ApJ, 812, L8

Walker G. A. H., Campbell E. K., Maier J. P., Bohlender D., Malo L., 2016, ApJ, 831, 130

Walker G. A. H., Campbell E. K., Maier J. P., Bohlender D., 2017, ApJ, 843, 56

Wang Y., Díaz-Tendero S., Alcamí M., Martín F., 2015, Nature Chem., 7, 927

Zhang Y., Kwok S., 2011, ApJ, 730, 126

Zhen J., Castellanos P., Paardekooper D. M., Linnartz H., Tielens A. G. G. M., 2014, ApJ, 797, L30

Zimmerman J. A., Eyler J. R., Bach S. B., McElvany S. W., 1991, J. Chem. Phys., 94, 3556

This paper has been typeset from a $\mathrm{TEX}_{\mathrm{E}} \mathrm{L} \mathrm{LT}_{\mathrm{E}} \mathrm{X}$ file prepared by the author. 